\title{
Síndrome nefrótico congénito por mutación del gen de la nefrina. Caso clínico
}

\author{
MARTA AZÓCAR P. \\ 1. Unidad de Nefrología, Servicio de Pediatría, Hospital Luis Calvo Mackenna. \\ Departamento de Pediatría Oriente, Facultad de Medicina, Universidad de Chile.
}

\begin{abstract}
Congenital nephrotic syndrome due to mutation of nephrine gene. Clinical case

Congenital Nephrotic Syndrome (CNS) is defined as a corticoresistant nephrotic syndrome which appears in the first 90 days of life. NPHS1 gene mutations, codifying nephrine cause nearly $40 \%$ of the cases. Such a clinical case has not yet been described in Chile. Objetive: Describe a patient with CNS and his genetic study. Clinical case: Full-term 38 week male newborn, birthweight 2620 gr, height 48,5 cm, APGAR 9/10. Head circumf: 33 $\mathrm{cm}$. First child, well controlled pregnancy. 16 days after birth, he develops edema, massive proteinuria $(3,2 \mathrm{gr} /$ $\mathrm{dl})$, hypoalbuminemia $(0,79 \mathrm{mg} / \mathrm{dl})$ and hypercholesterolemia ( total cholesterol: $318 \mathrm{mg} / \mathrm{dl})$. Renal sonogram showed increase in size and echogenicity in both kidneys, and loss of corticomedullar differentiation. Renal biopsy showed diffuse mesangial glomeruloesclerosis. Genetic study for NPHS1 was performed through direct sequencing of 29 exons and adjacent regions of introns chromosome 19q13.1. Analysis disclosed C567X, homozygote mutation. Conclusions: The first case of Nephrine mutation causing CNS is described in Chile. The importance of genetic studies in these patients is highlighted for clinical decisionmaking.
\end{abstract}

(Key words: Nephrotic syndrom - congenital).

Rev Chil Pediatr 2011; 82 (5): 426-431

\section{RESUMEN}

El síndrome nefrótico congénito (SNC) se define como un síndrome nefrótico córticoresistente que aparece en los primeros 90 días de vida. Mutaciones en el gen NPHS1 que codifica a la nefrina son causantes de aproximadamente del $40 \%$ de los niños con SNC. En Chile no se ha descrito hasta ahora esta mutación asociada al cuadro clínico. Objetivo: Describir el caso de un paciente con SNC y su estudio genético. Caso clínico: Paciente de sexo masculino, RNT 38 semanas, PRN 2620 gr, TN 48,5 cm APGAR 9/10. CC: 33 cm. Embarazo controlado primer hijo. A los 16 días comienza con edema, proteinuria masiva (3,2 gr/dl), hipoalbuminemia (0,79 mg/dl) e hipercolesterolemia (colesterol total: $318 \mathrm{mg} / \mathrm{dl})$. Se descarta TORCH (-). La ecografía renal mostró un aumento de tamaño y de ecogenicidad de ambos riñones, pérdida de diferenciación corticomedular. La biopsia renal se informó como glomeruloesclerosis mesangial difusa. El estudio genético para NPHS1 se realizó por secuenciación directa de los 29 exones y las regiones adyacentes de los intrones en el cromosoma 19q13.1. El análisis demostró una mutación C567X, homocigoto. Conclusiones: Se describe el primer caso de mutación para Nefrina en un SNC chileno. Se destaca la importancia del estudio genético en estos pacientes debido a las implicancias en las decisiones clínicas y terapéuticas.

(Palabras clave: Síndrome nefrotico - congénito).

Rev Chil Pediatr 2011; 82 (5): 426-431

Trabajo recibido el 07 de julio de 2011, devuelto para corregir el 16 de septiembre de 2011, segunda versión el 21 de septiembre de 2011, aceptado para publicación el 22 de septiembre de 2011.

Correspondencia a:

Marta Azócar P.

E-Mail: martitaazocar@gmail.com 


\section{Introducción}

El síndrome nefrótico congénito (SNC) se define como el síndrome nefrótico (SN) que aparece durante los 3 primeros meses de vida. En su gran mayoría cortico-resistentes, el 40$56 \%$ de estos pacientes presentan una mutación en el gen de la nefrina ${ }^{1,2}$. Este gen fue mapeado en 1994 en el cromosoma 19q13 y se describieron mutaciones en una población de niños afectados en 1998, contiene $26 \mathrm{~kb}$ en el ADN genómico y 29 exones, se denominó NPHS1 ${ }^{2}$ y su descripción constituyó uno de los grandes pasos en el campo del síndrome nefrótico (SN) hereditario. La nefrina es una proteína integral de membrana que pertenece a la superfamilia de las inmunoglobulinas 3,4, presenta un dominio transmembrana con 8 porciones tipo Ig hacia el extracelular, una porción tipo fibronectina III y un $\mathrm{N}$ - terminal intracelular; tiene forma de cierre y se expresa exclusivamente en los podocitos a nivel del diafragma de filtración glomerular(DFG); homodímeros y heterodímeros de la nefrina (figura 1), junto con la proteína glomerular $N E P H 1$ constituyen la base estructural del $\mathrm{DFG}^{2,3}$. El C-terminal extracelular une los espacios intercelulares entre los pedicelos, formando poros de aproximadamente de $40 \mathrm{~nm}$. Además de su rol como proteína estructural, la nefrina también participa en las señales intracelulares, manteniendo la funcionalidad integral del podocito.

Las mutaciones en NPHS1 se identificaron inicialmente en pacientes con SNC tipo finés ${ }^{1}$, que tiene un patrón de herencia recesiva y que clínicamente se caracteriza por proteinuria detectable desde el período intrauterino hasta dentro del primer año de vida. La placenta es de gran tamaño y a la histología renal los túbulos proximales muestran clásicamente dilatación microquística.

El SNC se observa en todo el mundo, pero es especialmente común en población finlandesa, donde 2 mutaciones fundadoras están frecuentemente presentes: deleción de dos pares de bases en el exón 2 (Fin mayor: p.L41fsX91), y una mutación nonsense en el exon 26 (Fin menor: p.R1109X). Hasta ahora más de 165 mutaciones distintas han sido identificadas, de estas la mayoría en pacientes no finlandeses, las cuales incluyen: nonsense, missense, frameshift inserción/deleción, y mutaciones de splicing que abarcan el gen completo $^{5-16}$. Investigaciones recientes confirman la existencia de una gran heterogeneidad genética y clínica en el SN asociado a mutaciones de NPHS1. El espectro de fenotipo asociado a mutaciones NPHS1 ha aumentado dramáticamente en los últimos años, debido a la identificación de mutaciones en la nefrina que causan enfermedad en pacientes que se presentan con SN en la infancia tardía.

En el reporte del NAPRTCS17 (North American Pediatric Renal Transplant Cooperative Study) publicado en el 2003 se reclutó 1992 niños en su registro de una cohorte de niños que iniciaban por primera vez HD o PD entre los años 1992 y 1998. De éstos, el 2,5\% tenía como diagnóstico etiológico un SNC ( $\mathrm{n}=$ 49). No existen registros disponibles en nuestro país ni en Latinoamérica de esta enfermedad.

\section{Objetivo}

Presentar un caso de SNC de causa genética en el cual se confirma una mutación para Nefrina, y destacar la relevancia clínica del estudio molecular.

\section{Caso clínico}

LCL, paciente varón, RNT 38 semanas PN 2620 g, TN 48,5 cm, APGAR 9/10. Parto cesárea por podálica. Embarazo controlado primer hijo. A los 16 días comienza con edema, proteinuria masiva (3,2 gr/dl), hipoalbuminemia $(0,79 \mathrm{mg} / \mathrm{dl})$, hipercolesterolemia (318 $\mathrm{mg} / \mathrm{dl}$ ) y creatininemia de $0,3 \mathrm{mg} / \mathrm{dl}$.

Se descartó TORCH. La ecografía mostró un aumento de tamaño renal para la edad y de ecogenicidad de ambos riñones, pérdida de diferenciación corticomedular.

Una primera biopsia renal realizada a los 2 meses de vida mostró celularidad glomerular normal,promedio de 95 células con acentuación de los ejes mesangiales lobulillares, el mesangio se apreció ensanchado y en dos glomérulos se observó una pequeña adherencia glomerulocapsular fibrosa con lesión seg- 


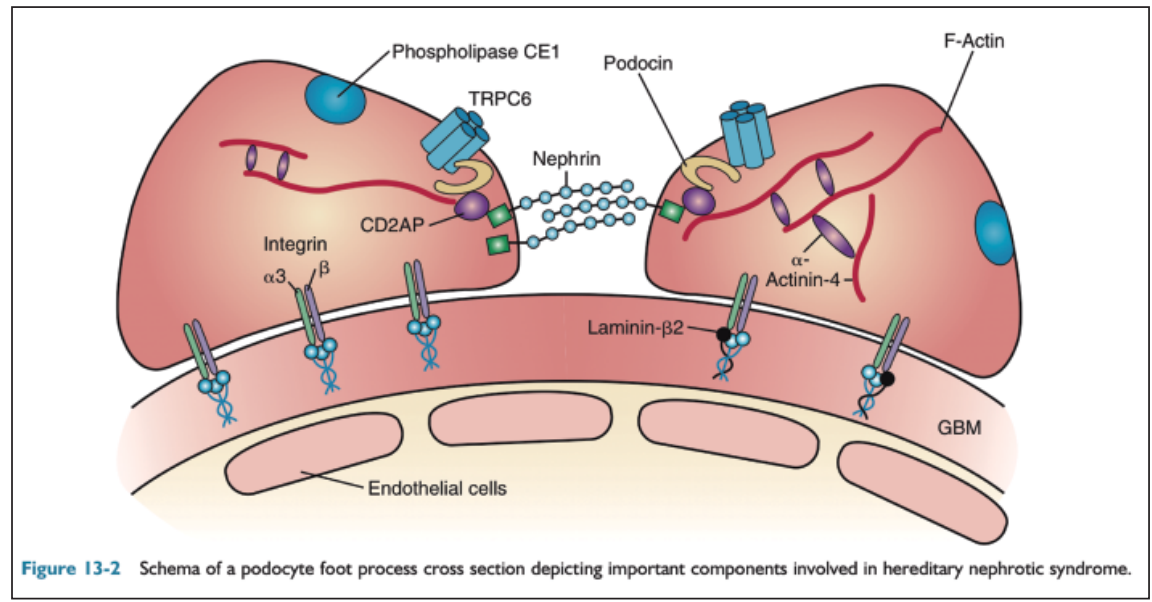

Figura 1. Referencia: Weber S. Hereditary nephrotic syndrome, in Comprehemsive Pediatric Nephrology, edited by Geary D. and Schaefer F. 2008; Elsevier 219-28. mentaria glomerular. En los túbulos se encontró signos de proteinuria y cilindros hialinos en moderada cantidad; el intersticio presentaba infiltrado focal linfoplasmocitario. Las arteriolas mostraron hiperplasia de miocélulas y leve estenosis del lumen. Se informó como alteraciones morfológicas compatibles con glomeruloesclerosis mesangial difusa. La microscopía electrónica mostró la membrana basal muy irregular con ensanchamientos a expensas de la lámina rara interna, con una lámina densa relativamente delgada; los podocitos tienen desaparición completa de pedicelos. La célula endoltelial está ligeramente tumefacta y tiene extensas áreas con desaparición de su lámina fenestrada.

Recibió tratamiento con prednisona $60 \mathrm{mg} /$ $\mathrm{m}^{2}$ /día por 30 días sin respuesta, evolucionó con deterioro de la función renal, creatinina: 2,68 mg/dl, y BUN 46 mg/dl. Destacaba oliguria y edema progresivo llegando a anasarca, hipertensión arterial, hipotiroidismo, hiperparatiroidismo secundario, e hipocalcemia severa. A los 3 meses de vida se decidió realizar nefrectomía izquierda, iniciando Diálisis Peritoneal con estabilización clínica, persistiendo con proteinuria masiva. A los 4 meses se realizó nefrectomía derecha ante persistencia de proteinuria masiva. El diagnóstico histológico de la pieza operatoria mostró microquistes tubulares, alteraciones mínimas glomerulares, compatible con síndrome nefrótico tipo finés.

A la edad de 11 meses se trasplantó con donante vivo, madre, recibiendo tratamiento inmunosupresor en base a ciclosporina, micofenolato mofetil y prednisona, normalizando función renal y sin recurrencia del síndrome nefrótico, con una evolución clínica satisfactoria.

\section{Estudio genético}

El estudio de la secuenciación genética fue realizado en el laboratorio de nefrología infantil Universidad de Heidelberg. El análisis del gen NPHS1 se realizó por secuenciación directa de los 29 exones y las regiones adyacentes de los intrones en el cromosoma 19q13.1. Se demostró mutación homocigota para el gen de la nefrina, C567X. Se realizó secuenciación genetica de WT1, cuyo resultado fue negativo para mutaciones. No se realizó estudio de otras mutaciones.

\section{Discusión}

$\mathrm{Al}$ enfrentar a un paciente lactante menor portador de un Síndrome nefrótico, es importante diferenciar si se trata de un SNC secundario o de causa genética. Dentro de las causas secundarias se describen infecciones congénitas como sífilis, toxoplasmosis o citomegalovirus, toxinas y síndrome hemolítico urémico, entre otros. De complejo manejo debido a la masiva pérdida de proteínas, estos paciente requieren infusiones de albúmina, nutrición 
parenteral, uso de inmunoglobulinas, prolongadas hospitalizaciones, en ocasiones nefrectomía, diálisis y trasplante renal, y presentan un alto riesgo de mortalidad. Clásicamente se describe resistencia al tratamiento con esteroides e inmunosupresores, y la enfermedad renal terminal se alcanza antes de los 2-3 años ${ }^{18}$.

El SNC tipo finés es una enfermedad autosómica recesiva secundaria a una mutación en la nefrina, caracterizada por proteinuria masiva y con ello el desarrollo de SN poco después del nacimiento. Esta enfermedad es frecuente en Finlandia, pero se ha identificado igualmente en otras poblaciones. En la población finlandesa 2 mutaciones han sido principalmente encontradas, correspondiendo aproximadamente al 94\% de las mutaciones en población Finlandesa. La mayoría de las mutaciones en pacientes de población no-finlandesa son mutaciones missense, pero también se han descrito mutaciones nonsense, splice site, deleciones e insersiones ${ }^{5-16}$.

En el estudio de Philippe et $\mathrm{al}^{19}$ se encontraron mutaciones en NPHS1 en los que la enfermedad se iniciaba desde los 6 meses hasta los 8 años, en tanto otros autores han encontrado mutaciones heterocigotas compuestas (p.R827X y p.R979S) en pacientes con inicio en edad adulta de desórdenes glomerulares ${ }^{20}$. Probablemente mutaciones de nefrina que son retenidas en el retículo endoplasmático, secundario a misfolding podrían explicar el inicio precoz del SN en pacientes ${ }^{21,22}$, mientras que una mutación leve permite una mantención parcial de la función proteica en los casos de inicio más tardíos del SN. También se ha reportado la presencia simultánea de mutaciones NPHS1 y NPHS2 ${ }^{9,23}$, pero hasta el momento no se dispone de evidencia de herencia oligogénica en casos de SNCR. Esta observación hace destacar un link funcional entre nefrina y podocina lo cual es apoyado por estudios celulares de su interacción ${ }^{24}$. Por otro lado, se han reportado idénticas mutaciones de la nefrina que producen distintos fenotipos desde inicio precoz hasta tardío del $\mathrm{SN}^{16,18,25}$, lo que sugiere que modificaciones en genes o factores ambientales podrían estar influyendo en la presentación y pronóstico de la enfermedad renal.

Desde el punto de vista histológico las biopsias de pacientes con SNC muestra lesiones mixtas glomerulares y túbulo intersticiales. Las lesiones glomerulares van desde enfermedad de cambios mínimos, hipercelularidad mesangial hasta glomeruloesclerosis focal y segmentaria y/o esclerosis mesangial difusa ${ }^{17,26}$. En el túbulo se describen característicamente dilataciones quísticas proximales y distales (conocidos como microquistes); inflamación y fibrosis intersticial están frecuentemente presentes y van aumentando con la edad. La inmunofluorescencia es negativa y la microscopía electrónica muestra desaparición de los pedicelos de los pococitos y transformación vellositaria. La tinción de nefrina puede darnos el diagnóstico definitivo en los casos de mutaciones truncadas, debido a que el diafragma de filtración glomerular está compuesto principalmente por nefrina y se encuenta ausente en glomérulos no esclerosados. Esto es especialmente importante para el diagnóstico diferencial en el SNC de otras causas ${ }^{3,17,26}$.

Desde un punto de vista clínico, esta enfermedad se describe como resistente al tratamiento con esteroides e inhibidores de la calcineurina, requiere frecuentes infusiones de albúmina y nutrición parenteral entre otras medidas para un manejo de sostén. Generalmente progresa rápidamente y alcanza la enfermedad renal crónica terminal en la primera década de la vida.

En el caso descrito en esta comunicación, el paciente no respondió a corticoides ni a la ciclosporina, lo que coincide con lo descrito en la literatura ${ }^{3,26,27}$.

Patrakka y cols ${ }^{28}$, describió un paciente portador de una mutación con un heterocigoto compuesto y una mutación missense, que respondió al tratamiento con indometacina e inhibidores de la enzima convertidora de la angiotensina. Otros 4 casos con mutaciones missense asociados a proteinuria recurrente/remitente han sido reportados en Japon ${ }^{29}$ y Alemania ${ }^{30}$. Philippe y cols ${ }^{19}$, describen fenotipos leves, con un buen pronóstico en 6 pacientes portadores de al menos una mutación severa, la cual en la mayoría de los casos derteminaban una proteína truncada. En el seguimiento clínico se observó un efecto antiproteinúrico del corticoide sólo o en asociación con ciclos- 
porina, y un paciente presentaba función renal normal a los 6 años de edad a pesar de no tener terapia. En el caso presentado, la mutación era homocigota y no se observó respuesta a los inmunosupresores. Esta mutación es conocida como una mutación causante de enfermedad.

\section{Conclusiones}

Durante las dos últimas décadas han sido cada vez más utilizados los estudios de búsqueda de mutaciones en la práctica clínica. En Chile, su utilización ha sido mayoritariamente en el área de la investigación, pero en los últimos años se ha difundido su utilidad entre la comunidad médica y entre los pacientes, cada vez mejor informados, con disponibilidad de buscar en internet información sobre su patología, aumentando la frecuencia con que los exámenes genéticos son solicitados.

El uso de técnicas moleculares en las formas genéticas de SNC permite un seguimiento especial en el paciente durante los primeros años de vida. Es importante que los médicos que puedan tener contacto con estos pacientes estén familiarizados con la existencia de las formas genéticas de SNC.

\section{Referencias}

1.- Kestila M, Lenkkeri U, Mannikko M, et al: Positionally cloned gene for a novel glomerular protein-nephrin-is mutated in congenital nephrotic syndrome. Mol Cell 1998; 1: 575-82.

2.- Pollak M: Inherited Podocytopathies: GEFS and nephrotic syndrome from a genetic viewpoint. J Am Soc Nephrol 2002; 13: 16-23.

3.- Weber S: Genetik des nephrotischen Syndroms. Der Nephrologe 2008; 5: 394-407.

4.- Benoit G, Machuca E, Heidet L, Antignac C: Hereditary kidney diseases: highlighting the importance of classical Mendelian phenotypes. Ann NY Acad Sci 2010; 1214: 83-98.

5.- Aya K, Tanaka H, Seino Y: Novel mutation in the nephrin gene of a Japanese patient with congenital nephrotic syndrome of the Finnish type. Kidney Int 2000; 57: 401-4.

6.- Beltcheva O, Martin P, Lenkkeri U, Tryggvason K: Mutation spectrum in the nephrin gene (NPHS1) in congenital nephrotic syndrome. Hum Mutat 2001; 17: 368-73.

7.- Gigante M, Greco P, Defazio V, et al: Congenital nephrotic syndrome of Finnish type: detection of new nephrin mutations and prenatal diagnosis in an Italian family. Prenat Diagn 2005; 25: 407-10.

8.- Gigante M, Monno F, Roberto R, et al: Congenital nephrotic syndrome of the Finnish type in Italy: a molecular approach. J Nephrol 2002; 15: 696-702.

9.- Koziell A, Grech V, Hussain S, et al: Genotype/phenotype correlations of NPHS1 mutations in nephrotic syndrome advocate a functional inter-relationship in glomerular filtration. Hum Mol Genet 2002; 11: 379-88.

10.- Sako M, Nakanishi K, Obana M, et al: Analysis of NPHS1, NPHS2, ACTN4, and WT1 in Japanese patients with congenital nephrotic syndrome. Kidney Int 2005; 67: 1248-55.

11.- Shih NY, Li J, Karpitskii V, et al: Congenital nephrotic syndrome in mice lacking CD2-associated protein. Science 1999; 286: 312-5.

12.- Frishberg Y, Ben-Neriah Z, Suvanto M, et al: Mis-leading findings of homozygosity mapping resulting from three novel mutations in NPHS1 encoding nephrin in a highly inbred community. Genet Med 2007; 9: 180-4.

13.- Hinkes BG, Mucha B, Vlangos $C N$, et al: Nephrotic syndrome in the first year of life: two thirds of cases are caused by mutations in 4 genes (NPHS1, NPHS2, WT1, and LAMB2). Pediatrics 2007; 119: e907-19.

14.- Heeringa SF, Vlangos CN, Chernin G, et al: Thirteen novel NPHS1 mutations in a large cohort of children with congenital nephrotic syndrome. Nephrol Dial Transplant 2008; 23: 3527-33.

15.- Ismaili K, Pawtowski A, Boyer O, Wissing KM, Janssen $F$, Hall ME: Genetic forms of nephrotic syndrome: a single-center experience in Brussels. Pediatr Nephrol 2009; 24: 287-94.

16.- Machuca E, Benoit G, Nevo F, et al: Genotype-phenotype correlations in non-Finnish congenital nephrotic syndrome. J Am Soc Nephrol 2010; 21: 1209-17.

17.- Leonard M, Donaldson L, Ho M, Geary D: A prospective cohort study of incident maintenance dialysis in children: An NAPRTC study. Kidney Int 2003; 63: 744-55.

18.- Schoeb D, Chernin G, Heeringa S, et al: Nineteen novel NPHS1 mutations in a worldwide cohort of patients with congenital nephrotic syndrome (CNS). Nephrol Dial Transplant 2010; 25: 2970-6.

19.- Philippe A, Nevo F, Esquivel EL, et al: Nephrin mutations can cause childhood-onset steroid-resistant nephrotic syndrome. J Am Soc Nephrol 2008; 19: 1871-8. 
20.- Santin S, García-Maset R, Ruiz P, et al: Nephrin mutations cause childhood- and adult-onset focal segmental glomerulosclerosis. Kidney Int 2009; 76: 1268-76.

21.- Liu L, Done SC, Khoshnoodi J, et al: Defective nephrin trafficking caused by missense mutations in the NPHS1 gene: insight into the mechanisms of congenital nephrotic syndrome. Hum Mol Genet 2001; 10: 2637-44.

22.- Roselli S, Moutkine I, Gribouval O, Benmerah A, Antignac $C$ : Plasma membrane targeting of podocin through the classical exocytic pathway: effect of NPHS2 mutations. Traffic 2004; 5: 37-44.

23.- Lowik M, Levtchenko E, Westra D, et al: Bigenic heterozygosity and the development of steroid-resistant focal segmental glomerulosclerosis. Nephrol Dial Transplant 2008; 23: 3146-51.

24.- Huber TB, Kottgen M, Schilling B, Walz G: Benzing. Interaction with podocin facilitates nephrin signaling. J Biol Chem 2001; 276: 41543-6.

25.- Coulthard MG: Manegement of Finnish congenital nephritic syndrome by unilateral nephrectomy. Pediatr Nephrol 1989; 3: 451-53.

26.- Caridi G, Trivelli A, Sanna-Cherchi S, Perfumo F, Ghiggeri $G$ : Familial forms of nephrotic syndrome. Pediatr Nephrol (2010) 25: 241-52.

27.- Weber S: Hereditary nephrotic syndrome, in Comprehesive Pediatric Nephrology, edited by Geary D. and Schaefer F. 2008. Elsevier. 219-28.

28.- Patrakka J, Kestila M, Wartiovaara J, et al: Congenital nephrotic syndrome (NPHS1): features resulting from different mutations in Finnish patients. Kidney Int 2000; 58: 972-80.

29.- Kitamura A, Tsukaguchi H, Hiramoto R, et al: A familial childhood-onset relapsing nephrotic syndrome. Kidney Int 2007; 71: 946-51.

30.- Heeringa SF, Vlangos CN, Chernin G, et al: Thirteen novel NPHS1 mutations in a large cohort of children with congenital nephrotic syndrome. Nephrol Dial Transplant 2008; 23: 3527-33. 